\title{
Influenza in the Elderly - A Mini-Review
}

\author{
A. Pop-Vicas ${ }^{\text {a }}$ S. Gravenstein ${ }^{b}$ \\ a Division of Infectious Diseases, Memorial Hospital of Rhode Island, Alpert Medical School at Brown University, \\ Pawtucket, R.I., and ${ }^{b}$ Division of Geriatrics, Alpert Medical School at Brown University, Providence, R.I., USA
}

\section{Key Words}

Influenza $\cdot$ Influenza vaccine

\begin{abstract}
Influenza is an important cause of morbidity and mortality in the elderly population each year. The often subtle clinical manifestations in the frail geriatric patients may not be recognized initially, impeding timely administration of antiviral treatment. The effectiveness of current influenza vaccines in the elderly population is often diminished by immune senescence. Increasing immunization rates among health-care workers and elderly caregivers, and finding more effective vaccines for the elderly people are likely to significantly improve disease prevention in this population at risk.
\end{abstract}

Copyright $\odot 2010$ S. Karger AG, Basel

\section{Introduction}

Influenza remains the leading cause of infectious death among elderly people, largely due to declining immune competence with age. It is also the most important agent of outbreaks of respiratory illness. Influenza continues to be important despite advances in its prevention and control due to the unique ability of the virus to undergo frequent antigenic variation. Minor changes in the structure of the surface glycoproteins, hemagglutinin and neuraminidase (antigenic drift) allow for yearly, typically winter, epidemics. Major changes (antigenic shift) occur less frequently and are associated with worldwide pandemics.

\section{Epidemiology and Disease Burden}

Seasonal influenza, directly or indirectly, causes approximately 36,000 deaths each year [1] and 226,000 hospitalizations [2]. The risk of influenza-related death increases exponentially after the age of 65 , with elderly people incurring more than $90 \%$ of overall influenza-related mortality annually [1]. Not just age, but the presence of underlying comorbid conditions further increases the risk of death from influenza. Nursing-home residents are most susceptible to influenza, given their multimorbidity and greater exposure risk through close living quarters and shared caregivers. Frailty and nutritional deficiencies may exacerbate their vulnerability to disease even more.

Influenza can produce significant functional decline in elderly patients [3]. In general, over one third of hospitalized patients age 70 or older leave the hospital more disabled than when they arrived [4]. Since influenza-related hospitalizations are more frequent and prolonged among elderly patients, influenza likely contributes significantly to this functional loss.

In contrast to seasonal influenza, the majority of symptomatic infections during pandemic influenza occur among young adults. Experts attribute this relative sparing of older adults to persistent immunological memory from prior exposure to similar viruses decades earlier. Indeed, 34\% of the adults born before 1950 had crossreactive antibodies to the pandemic $\mathrm{H} 1 \mathrm{~N} 1$ virus at the beginning of the 2009 pandemic [5]. However, these data should be considered preliminary, as the differences in symptom presentation may not be due to better viremic

\section{KARGER}

Fax +4161306 1234

E-Mail karger@karger.ch

www.karger.com
(C) 2010 S. Karger AG, Basel

0304-324X/11/0575-0397\$38.00/0

Accessible online at:

www.karger.com/ger
Aurora Pop-Vicas, MD, MPH

Division of Infectious Diseases, Memorial Hospital of Rhode Island

Alpert Medical School at Brown University

111 Brewster Street, Pawtucket, RI 02860 (USA)

Tel. +1 401729 2545, Fax +1 401729 2795,E-Mail Aurora_Pop-Vicas@brown.edu 
control in elderly individuals, but a more powerful innate immune response in the younger individuals.

Although disproportionately fewer elderly people developed clinical influenza, their mortality during the 2009 H1N1 pandemic remained substantial. For example, an analysis of patients hospitalized with pandemic H1N1 virus in California found that persons $\geq 50$ years of age had the highest influenza fatality rate (18-20\%) [6]. As with seasonal influenza, the presence of underlying chronic diseases increased the mortality risk [6].

\section{Transmission and Viral Shedding}

Influenza is transmitted primarily through droplet aerosols generated by coughing, sneezing, or talking [7]. Airborne transmission may occur in health-care settings during aerosol-generating procedures, such as intubation or mechanical ventilation.

In the absence of antiviral treatment, viral shedding typically starts within $24 \mathrm{~h}$ before the onset of symptoms, and continues for approximately 5 days in healthy adults [8]. In elderly adults, especially those with significant comorbidities, immunocompromise, or ill enough to require mechanical ventilation, viral shedding can last significantly longer [9]. Shedding can be significantly reduced by the use of antiviral medications within the first 48-96 h of illness [9], and may be prevented altogether with chemoprophylaxis [10].

\section{Pathogenesis and Host Defense}

After inhalation, virus particles attach to the ciliated columnar epithelial cells of the respiratory tract. The mechanical defense of the respiratory tract includes mucociliary clearance and cough, both of which are often impaired in older age, and antihemagglutinin mucosal IgA secretory antibodies. Viral replication is also inhibited by type I interferon production from natural killer cells and viral-infected host cells, and these cellular events seem to correlate with the clinical presence of fever. Animal studies suggest that fever generation may accelerate viral clearance, whereas fever suppression may be associated with an increase in the amount of nasal viral shedding [11]. The reduced prevalence of fever with advancing age may help explain why older people shed virus longer than their younger counterparts, presenting less symptomatically, even with potentially deadly illnesses.

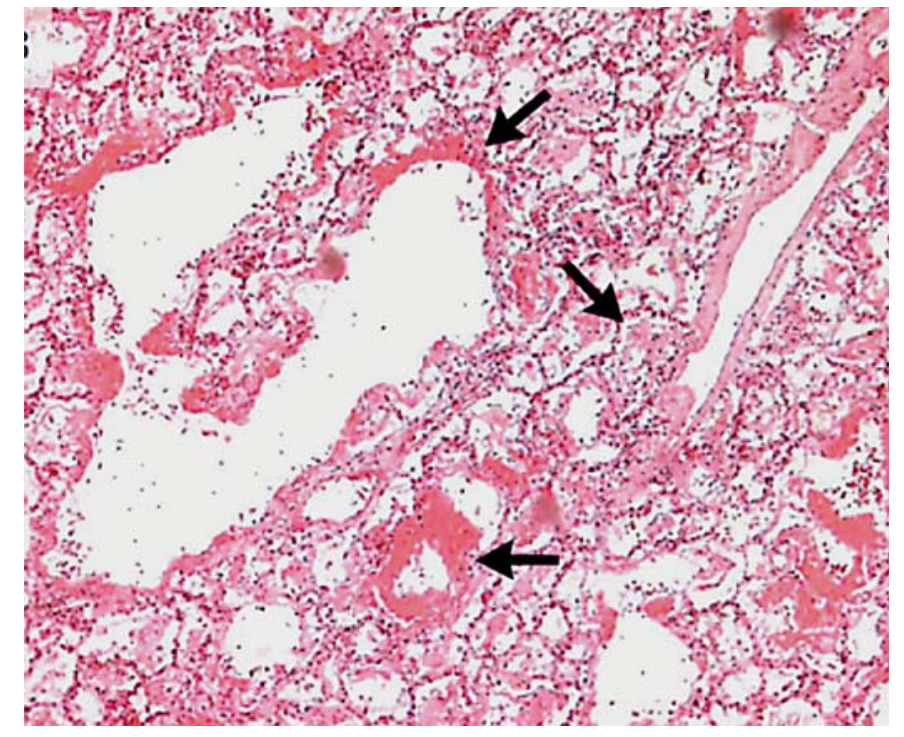

Fig. 1. Hematoxylin and eosin stain of lung tissue from a patient who ultimately died of H1N1 influenza pneumonia. Note necrosis of bronchiolar walls (top arrow), a neutrophilic infiltrate (middle arrow), and diffuse alveolar damage with prominent hyaline membranes (bottom arrow) [reproduced with permission from 54].

Serum $\operatorname{IgG}$ antibodies are important in protecting against lower respiratory tract infection and pneumonia. Host antibody responses are strain-specific, and antibodies are absent in individuals without previous exposure to the particular infecting strain or its antigens. Therefore, the propensity of influenza viruses to mutate frequently necessitates the recommended annual vaccinations to help maintain adequate influenza immunity.

In the absence of the above-mentioned host defenses, viral replication and cell death continue, propagating epithelial injury of the respiratory tract. Electron-microscopic images from experimentally infected mice show complete tracheal epithelial desquamation after the first 3 days of infection, and epithelial regeneration in the following days [12]. In fatal pandemic $2009 \mathrm{H} 1 \mathrm{~N} 1$ infections, the amount of lung damage has been extensive, evidenced by hyaline membranes, alveolar hemorrhages, and necrotizing bronchiolitis at autopsy [13] (fig. 1). Aberrant immune responses and hyperproduction of inflammatory cytokines such as interferon- $\gamma$ and TNF- $\alpha$ also frequently lead to fatal influenza [13].

As humoral responses interfere with infection and viral propagation, cell-mediated immune responses, particularly cytotoxic $\mathrm{T}$ lymphocytes, contribute to recovery from infection. Cellular responses promote viral clearing 
and also help protect against pneumonia. Because many cytotoxic T lymphocytes respond to peptides from evolutionarily conserved, internal viral proteins, they can be cross-protective against different influenza A strains [14]. Therefore, during pandemic scenarios, when there is little protective humoral immunity in the population, residual cytotoxic $\mathrm{T}$ lymphocyte immunity can play a significant protective role [15].

\section{Influenza Immunity and Aging}

The mechanisms responsible for immune senescence and their relevance to influenza infection in the elderly have not been fully elucidated, and a detailed review is beyond the scope of this paper. Some of the age-associated changes in immunity and their relevance to influenza are summarized below.

\section{Innate Immunity}

Toll-like receptors are evolutionarily conserved molecules, which recognize a broad array of antigens. They are expressed on antigen-presenting cells during an infection, and contribute to the stimulation of the proinflammatory cascade, with the subsequent activation of specific adaptive immunity responses. Experiments in aged mice have shown a decline in the expression of Tolllike receptors on macrophages, and a decrease in the amount of proinflammatory cytokines produced upon antigenic stimulation with influenza compared with young mice [16]. Similarly, older people have fewer plasmacytoid dendritic cells, and produce less interferon- $\alpha$ to stimulate downstream innate and adaptive immune mechanisms [17]. The ensuing muted inflammatory response with reduced interferon and interleukin production helps explain the milder initial clinical presentation of disease, the reduced prevalence of fever in elderly patients, and possibly the reduced vaccine responsiveness in others [18].

\section{Adaptive Immunity}

The ability to mount effective humoral responses to new antigens declines with aging. Antibody titers in response to influenza vaccines are often lower in elderly people compared to young adults [19]. Cell-mediated immune responses also decline with age [20], and this may explain why influenza lasts longer in elderly patients. These changes with age likely result from both the normal age-related thymic involution, as well as thymus-independent cell-level changes occurring with antigen-pre- senting and other immune cells. Collectively, these changes leave elderly people more vulnerable to the effects of influenza and other infections, and less able to mount a protective response to influenza immunizations.

\section{Clinical Manifestations}

\section{Uncomplicated Influenza}

The incubation period is usually $24-48 \mathrm{~h}$. The classical symptoms of influenza include the abrupt onset of fever, chills, myalgias, headache, and malaise. Fever usually lasts 3 days, although in some cases it may persist up to 1 week. Respiratory symptoms, such as dry cough, severe sore throat, and nasal discharge, become more evident as the initial systemic symptoms begin to subside. Physical exam may reveal hyperemic mucous membranes, small, tender cervical lymphadenopathy, flushed face, and moist skin. The illness usually resolves in 5-7 days, although a convalescent period characterized by cough, lassitude, and malaise (postinfluenza asthenia) is fairly common, and may last for several weeks [7]. In older adults, the respiratory symptoms may initially be very subtle and difficult to recognize. Instead, elderly patients may present only with cough, fatigue, and confusion [21]. While children and younger adults can have fevers as high as 104$106^{\circ} \mathrm{F}$, the fever response may be more blunted in the older adults [7], and in nursing-home elderly influenza often fails to produce fever over $99^{\circ} \mathrm{F}$. The elderly patients are the age group most susceptible to pulmonary complications from influenza.

\section{Pulmonary Complications}

Primary Influenza Viral Pneumonia. Primary viral pneumonia represents the most severe pulmonary manifestation of influenza. Although unusual with seasonal influenza epidemics, it becomes prominent in influenza pandemics, and is responsible for much of the associated mortality [22]. The syndrome, characterized by a typical onset of influenza, rapidly progresses to severe respiratory distress. Laboratory and radiological evaluation often show marked hypoxemia and bilateral interstitial infiltrates typical of acute respiratory distress syndrome, in the absence of a bacterial pulmonary pathogen on Gram stain of the sputum. Nasopharyngeal or respiratory clinical specimens usually have high influenza viral titers. Autopsy findings are as noted above. During the current pandemic with the $2009 \mathrm{H} 1 \mathrm{~N} 1$ virus, as with previous influenza pandemics, primary influenza pneumonia so 
far has been more frequent in young, otherwise healthy adults, and rare in elderly patients.

Secondary Bacterial Pneumonia. Secondary bacterial pneumonia is a major source of influenza-related morbidity and mortality in the elderly. It is characterized by a biphasic illness, with initial typical influenza-type symptoms, a few days of clinical improvement, followed by recurrence of fever, cough with purulent sputum, and evidence of pulmonary consolidations on radiographic testing. The most frequent etiologic pathogens include Streptococcus pneumoniae, Staphylococcus aureus, and Haemophilus influenzae [7]. As with other infections in the aged, the clinical presentation of bacterial pneumonia is often atypical, affecting mental status, and reducing fever or pleuritic chest pain unlike that in younger adults [23]. Bacterial coinfections, although not recognized during the early phase of the $2009 \mathrm{H} 1 \mathrm{~N} 1$ influenza pandemic, played a significant role in the pandemic-associated mortality, being present in approximately one third of the fatal cases [24].

\section{Other Complications}

Exacerbation of chronic pulmonary disease, such as asthma or chronic bronchitis, frequently complicates influenza infections, and can further impair pulmonary function [7]. Mixed viral and bacterial pneumonias may also occur. Influenza-related cardiovascular morbidity, with outcomes of heart disease and stroke, while less recognized, is equally important [25]. Other rare complications, such as myositis, rhabdomyolysis, and encephalitis, have also been described [7].

\section{Diagnosis}

\section{Epidemiological Diagnosis}

The presence of fever and cough within $48 \mathrm{~h}$ of symptom onset has a $79 \%$ positive predictive value for influenza during a period of high influenza activity in the community, such as an influenza outbreak [26]. In the absence of an influenza outbreak, clinical manifestations alone are not useful in trying to distinguish influenza from other respiratory viral pathogens such as rhinovirus or coronavirus, and laboratory confirmation should be pursued.

\section{Rapid Antigen Tests}

Rapid antigen tests detect virus antigens in the sample. Samples are collected by swabbing the throat, anterior nares or nasopharyngeal mucosa, depending on the test and technician skill. The virus antigen may be free with- in the nasopharyngeal secretions or intracellular. The higher-quality samples have evidence of cellularity as judged by the turbidity of the sample. These tests have the advantage of being widely available in many health-care settings, offering results within minutes from testing. Unfortunately, their sensitivity for detecting both seasonal and pandemic influenza strains remains limited [27]. Therefore, a negative result on a rapid antigen test does not preclude the presence of either seasonal or pandemic 2009 H1N1 influenza, and should not be used for this purpose.

\section{Molecular Diagnostics}

Reverse transcriptase-polymerase chain reaction (RTPCR) has the advantage of being the most sensitive and specific diagnostic modality, with the ability to distinguish between different influenza types, including the diagnosis of the novel $2009 \mathrm{H} 1 \mathrm{~N} 1$ pandemic strain. Molecular diagnostics use samples collected similarly to those for rapid antigen tests. The RT-PCR is the recommended test modality for confirmation of the 2009 H1N1 pandemic strain [28]. Unfortunately, the test remains largely unavailable at the point of care, being primarily used in public health or research laboratories.

\section{Immunofluorescence}

Immunofluorescent antibody staining for influenza antigen detection has good sensitivity for the detection of both seasonal and pandemic influenza strains [29]. However, as this test requires considerable technical expertise and a fluorescent microscope, it is not widely available. Samples for immunofluorescence are collected in a similar fashion as those for rapid antigen testing. The test has greater sensitivity in samples that have greater cellularity.

\section{Viral Culture}

Although culture has been considered the gold standard for influenza diagnosis, the standard may move to molecular diagnostics in the next years. The time span of several days until culture results become available limits their clinical utility for initial clinical management. Samples suitable for rapid antigen testing can also be used for viral culture, and high cellularity increases the likelihood of viral growth. However, some test kits, like those for molecular diagnostics, include chemicals that stop RNA replication and would also interfere with growing the virus in culture. Viral culture remains a useful modality for confirmation of negative screening tests in cases where influenza is highly suspected and for surveillance [30]. 


\section{Serology}

Measures of antibody change over time provide a useful sensitive and specific tool to confirm the diagnosis of influenza retrospectively, but offer little value to the point of care practitioner.

\section{Treatment}

Among ambulatory patients, antiviral treatment initiated within the first $48 \mathrm{~h}$ of symptom onset can shorten the duration of symptoms and reduce influenza-related complications [30]. Among hospitalized patients, antiviral treatment has been shown to reduce mortality and decrease the length of hospitalization [31], and some benefit remains even if antivirals are started after the first $48 \mathrm{~h}$ of symptom onset [32]. The neuraminidase inhibitors are active against most strains of seasonal influenza $A$ and $\mathrm{B}$, and are the treatment of choice for the pandemic 2009 H1N1 influenza A strain. They are generally well tolerated, and are available in oral (oseltamivir) and inhaled powder (zanamivir) formulations. Peramivir, an unapproved investigational neuraminidase inhibitor, is available from the CDC under Emergency Use Authorization for cases of $2009 \mathrm{H} 1 \mathrm{~N} 1$ influenza that require intravenous antiviral administration [33]. Zanamivir may cause bronchospasm in patients with underlying reactive airway disease. Therefore, if the drug is administered to these patients, it should be done in conjunction with bronchodilators [7]. Oseltamivir has lost effectiveness against seasonal $\mathrm{H} 1 \mathrm{~N} 1$ influenza due to resistance in $98.5 \%$ of the seasonal H1N1 isolates from the 2008-2009 season in the US. Fortunately, these strains remain susceptible to zanamivir, amantadine, and rimantadine. During the 20092010 influenza season, the predominant strain in circulation was the 2009 pandemic H1N1 strain, which remains largely susceptible to neuraminidase inhibitors. Cases of oseltamivir resistance during the $2009 \mathrm{H} 1 \mathrm{~N} 1$ pandemic, still exceedingly rare, thus far have occurred mainly in immunocompromised patients with prolonged viral shedding, or in patients who previously received oseltamivir chemoprophylaxis [34]. The M2 inhibitors, amantadine and rimantadine, initially effective against influenza $\mathrm{A}$, are no longer recommended, as virtually all seasonal $\mathrm{H} 3 \mathrm{~N} 2$ viruses during recent years have become resistant to these agents. They are not active against influenza B or against the $2009 \mathrm{H} 1 \mathrm{~N} 1$ pandemic strain. Amantadine or rimantadine do provide protection for seasonal H1N1 influenza A and can be used in place of zanamivir for this indication [30] (table 1).

Influenza in the Elderly
Table 1. Antiviral treatment of influenza according to drug susceptibility of each influenza strain

\begin{tabular}{|c|c|c|c|c|}
\hline \multirow{2}{*}{$\begin{array}{l}\text { Antiviral } \\
\text { drug }\end{array}$} & \multicolumn{4}{|c|}{ Susceptibility to antiviral drug } \\
\hline & $\begin{array}{l}\text { pandemic } \\
\text { H1N1 }\end{array}$ & $\mathrm{H} 3 \mathrm{~N} 2$ & $\begin{array}{l}\text { seasonal } \\
\text { H1N1 }\end{array}$ & influenza B \\
\hline Oseltamivir & susceptible $^{1}$ & susceptible & resistant & susceptible \\
\hline Zanamivir & susceptible & susceptible & susceptible & susceptible \\
\hline Amantadine & resistant & resistant & susceptible & no activity \\
\hline Rimantadine & resistant & resistant & susceptible & no activity \\
\hline
\end{tabular}

1 Isolated reports of oseltamivir-resistant pandemic H1N1 strains have been reported in immunocompromised patients with prolonged viral shedding, or in patients who have received prior chemoprophylaxis with oseltamivir [34]. The majority of pandemic H1N1 strains remain susceptible to oseltamivir.

\section{Prevention}

Influenza vaccination offers the most cost-effective approach to influenza prevention. The National Advisory Committee on Immunization Practices recommends influenza vaccination for adults 50 years or older and their caregivers [35]. Among healthy elderly volunteers, the influenza vaccine proved to be $50 \%$ effective in reducing laboratory-confirmed influenza compared to a placebo group [36]. Although only $23 \%$ effective for elderly over 70 years of age, with a wide confidence interval including 0 , the small study sample size limits the confidence in the vaccine efficacy estimate for this population. A Cochrane review of all 5 randomized-control trials found a vaccine effectiveness of $58 \%$ against influenza and $43 \%$ against influenza-like illness [37]. Another systematic review of studies of influenza vaccine in the elderly, including data from observational trials, found a $26 \%$ reduction in hospitalization from influenza and pneumonia, and a $42 \%$ reduction in all-cause mortality among communitydwelling elderly [38]. Among nursing-home residents, well-matched vaccines were found to be $46 \%$ effective in preventing pneumonia, and $60 \%$ effective in reducing allcause mortality [38]. Vaccine-associated protective effects appear to increase with repeated vaccinations. The study by Ahmed et al. [39] reported less mortality in patients vaccinated more than once compared with patients vaccinated only once (75 vs. $9 \%$ ). Similarly, in a large population study among community-dwelling elderly followed during 1996 through 2002, annual influenza vaccinations were associated with a significant decrease in the risk of death. Mortality risk, however, increased to $40 \%$ above 
baseline if annual vaccination was interrupted, and returned to baseline after vaccine was reinitiated [40].

Despite these data, the effectiveness of influenza vaccination in reducing all-cause mortality in the elderly has been called into question in recent years. An ecological analysis studying the impact of influenza vaccination on seasonal mortality over 33 seasons, from 1968 to 2001, found that excess winter mortality among US elderly $\geq 65$ years of age never exceeded $10 \%$ of the total winter mortality. The authors argued, therefore, that the much larger mortality reductions observed repeatedly in observational studies is implausible, and must be the result of bias inherently associated with nonrandomized clinical studies [41]. The analysis also noted a paradoxical increase in all-cause mortality among the elderly people despite steady increases in vaccine coverage over the last decades [41].

However, these observations do not rise to the evidence of the randomized, placebo-controlled trials that establish efficacy. The above study also has been criticized for lack of individual data on vaccination status and other related individual health variables, as one of the major limitations of ecological analyses [42]. The increase in mortality has been explained by the increase in aging of the US population, and the increase in duration of annual influenza epidemics, from an average of 6 weeks during the 1970s to 16 weeks during the 1980 s [43].

From an immunological standpoint, concern over the effectiveness of influenza vaccine in the elderly also exists. Most studies agree that current vaccines are less immunogenic and less effective in the elderly population compared with younger, healthier adults. As presented above, this may be due, at least in part, to immune senescence, which impairs the ability to respond appropriately to newly encountered antigens.

All influenza vaccines currently licensed for use in the elderly patients are trivalent inactivated vaccines given intramuscularly. Intranasal live-attenuated influenza vaccines are not approved for use in elderly people. In light of the current limitations associated with influenza vaccines in this population, developing additional strategies for disease prevention in the elderly should be useful. In this regard, an alternate route of vaccine delivery - intradermal administration - was associated with increased immunogenicity in a study of patients older than 60 [44], although other studies have not confirmed these findings [45]. Adjuvanted influenza vaccines represent another potential strategy to increase immunogenicity in elderly patients. In a small study of long-term care facility resi- dents in Italy, an MF59-adjuvant influenza vaccine was more efficacious in preventing influenza-like illness compared with a nonadjuvanted vaccine [46]. The MF59adjuvanted influenza vaccine has been licensed in Europe since 1997. In a study comparing standard-dose vaccines with a high-dose inactivated influenza vaccine the latter has been shown to be more immunogenic in people older than 65 [47]. Based on these results, a high-dose inactivated influenza vaccine has been recently licensed in the US for individuals $\geq 65$ [48].

In addition to these investigational approaches, there is increasing evidence that immunizing children and younger adults, who respond well to current vaccines, would reduce the burden of influenza in the elderly by reducing their exposure risk. For example, a modeling study of the population-wide impact of influenza immunizations suggests that a vaccination rate of $20 \%$ among children aged 6 months to 18 years would reduce the total US cases of influenza-related hospitalizations and deaths by half [49]. A study comparing two Russian towns with different childhood immunization policies found that the rate of chronic disease exacerbations was much lower in the town with mass childhood immunization than in the town where childhood immunizations were not provided [50]. Similarly, immunizing school-age children in Japan was associated with substantial reduction in influenza-related mortality in their elderly population [51]. In long-term care facilities, vaccinating health-care workers has been associated with decreased mortality among nursing-home residents [52]. Improving the efficacy of current influenza vaccines for the elderly population, through dose increases, biologic response modifiers, or alternative modes of delivery, is an area of ongoing research.

With regard to the newly developed $\mathrm{H} 1 \mathrm{~N} 1$ vaccine, preliminary studies indicate that the vaccine is immunogenic and safe for both healthy adults and elderly people [53]. Therefore, the vaccine should be offered to the elderly population at risk. In light of the recently reported 2009 pandemic H1N1 influenza outbreaks among longterm care facility residents, timely vaccination of both residents and health-care workers against the $2009 \mathrm{H} 1 \mathrm{~N} 1$ strain becomes particularly important [43].

Antiviral chemoprophylaxis is another effective modality of influenza prevention. In the event of an institutional influenza outbreak, antiviral chemoprophylaxis is recommended for all residents of the affected longterm care facility, regardless of their vaccination status [30]. Chemoprophylaxis should also be considered for community-dwelling elderly at high risk for influenza- 
related complications, if they had not yet been immunized at the time of influenza circulation in their community [30].

\section{Conclusion}

Influenza, the leading cause of preventable infectious death in older adults, is still easily overlooked. Difficulties in diagnosing influenza due to altered presentation often preclude timely administration of antiviral therapy. Diminished vaccine responses in frail elderly people also undermine our ability to control this important disease. Nevertheless, influenza vaccines offer substantial reductions in hospitalizations and mortality, and our efforts to immunize the oldest patients, their health-care workers and caregivers, and other at-risk populations should increase. Finding new ways to overcome senescent immune responses and offer better vaccines for the elderly population is of utmost importance.

\section{References}

1 Thompson WW, et al: Mortality associated with influenza and respiratory syncytial virus in the United States. JAMA 2003;289: 179-186.

2 Thompson WW, et al: Influenza-associated hospitalizations in the United States. JAMA 2004;292:1333-1340.

- 3 Barker WH, Borisute H, Cox C: A study of 14 the impact of influenza on the functional status of frail older people. Arch Intern Med 1998;158:645-650.

4 Covinsky KE, et al: Loss of independence in activities of daily living in older adults hospitalized with medical illnesses: increased vulnerability with age. J Am Geriatr Soc 2003;51:451-458.

5 Hancock K, et al: Cross-reactive antibody responses to the 2009 pandemic H1N1 influenza virus. N Engl J Med 2009;361:19451952.

6 Louie JK, et al: Factors associated with death or hospitalization due to pandemic 2009 influenza A (H1N1) infection in California. JAMA 2009;302:1896-1902.

7 Treanor JJ: Influenza viruses, including avian influenza and swine influenza; in Mandell GL, Bennett JE, Dolin R (eds): Mandell, Douglas, and Bennett's Principles and Practice of Infectious Diseases. Philadelphia, Churchill Livingstone, 2009, pp 2265-2288.

$\checkmark 8$ Carrat F, et al: Time lines of infection and disease in human influenza: a review of volunteer challenge studies. Am J Epidemiol 2008; 167:775-785.

9 Lee N, et al: Viral loads and duration of viral shedding in adult patients hospitalized with influenza. J Infect Dis 2009;200:492-500.

10 Gravenstein S, et al: Inhaled zanamivir versus rimantadine for the control of influenza in a highly vaccinated long-term care population. J Am Med Dir Assoc 2005;6:359-366.

11 Husseini RH, et al: Elevation of nasal viral levels by suppression of fever in ferrets infected with influenza viruses of differing virulence. J Infect Dis 1982;145:520-524.
12 Ramphal R, et al: Murine influenzal tracheitis: a model for the study of influenza and tracheal epithelial repair. Am Rev Respir Dis 1979;120:1313-1424.

13 Mauad T, et al: Lung pathology in fatal novel human influenza A (H1N1) infection. Am J Respir Crit Care Med 2010;181:72-79.

14 Yewdell JW, et al: Influenza A virus nucleoprotein is a major target antigen for crossreactive anti-influenza A virus cytotoxic $\mathrm{T}$ lymphocytes. Proc Natl Acad Sci USA 1985; 82:1785-1789.

15 McMichael AJ, et al: Cytotoxic T-cell immunity to influenza. N Engl J Med 1983;309: 13-17.

16 Renshaw M, et al: Cutting edge: impaired Toll-like receptor expression and function in aging. J Immunol 2002;169:4697-4701.

17 Deng Y, et al: Age-related impaired type $1 \mathrm{~T}$ cell responses to influenza: reduced activation ex vivo, decreased expansion in CTL culture in vitro, and blunted response to influenza vaccination in vivo in the elderly. J Immunol 2004;172:3437-3446.

18 McElhaney JE: Prevention of infectious diseases in older adults through immunization: the challenge of the senescent immune response. Expert Rev Vaccines 2009;8:593606.

19 Saurwein-Teissl M, et al: Lack of antibody production following immunization in old age: association with CD8(+)CD28(-) T cell clonal expansions and an imbalance in the production of Th1 and Th 2 cytokines. J Immunol 2002;168:5893-5899.

20 McElhaney JE, et al: Immune response to influenza vaccination in institutionalized elderly: effect on different T-cell subsets. Vaccine 1998;16:403-409.

21 Walsh EE, Cox C, Falsey AR: Clinical features of influenza A virus infection in older hospitalized persons. J Am Geriatr Soc 2002; 50:1498-1503.

22 Louria DB, et al: Studies on influenza in the pandemic of 1957-1958. II. Pulmonary complications of influenza. J Clin Invest 1959; 38(1 Part 2):213-265.
23 Bentley DW: Bacterial pneumonia in the elderly: clinical features, diagnosis, etiology, and treatment. Gerontology 1984;30:297307.

24 Centers for Disease Control and Prevention (CDC): Bacterial coinfections in lung tissue specimens from fatal cases of 2009 pandemic influenza A (H1N1) - United States MayAugust 2009. MMWR Morb Mortal Wkly Rep 2009;58:1071-1074.

25 Nichol KL, et al: Influenza vaccination and reduction in hospitalizations for cardiac disease and stroke among the elderly. N Engl J Med 2003;348:1322-1332.

26 Monto AS, et al: Clinical signs and symptoms predicting influenza infection. Arch Intern Med 2000;160:3243-3247.

27 Faix DJ, Sherman SS, Waterman SH: Rapidtest sensitivity for novel swine-origin influenza A (H1N1) virus in humans. N Engl J Med 2009;361:728-729.

28 United States Center for Disease Control and Prevention: Interim guidance on specimen collection, processing, and testing for patients with suspected swine-origin influenza A (H1N1) virus infection. 2009. http://www. cdc.gov/h1n 1 flu/specimencollection.htm (accessed July 24, 2009).

29 Pollock NR, et al: Ruling out novel H1N1 influenza virus infection with direct fluorescent antigen testing. Clin Infect Dis 2009; 49:e66-e68.

30 Harper SA, et al: Seasonal influenza in adults and children - diagnosis, treatment, chemoprophylaxis, and institutional outbreak management: clinical practice guidelines of the Infectious Diseases Society of America. Clin Infect Dis 2009;48:1003-1032.

- 31 Webb SA, et al: Critical care services and 2009 H1N1 influenza in Australia and New Zealand. N Engl J Med 2009;361:1925-1934.

-32 Ison MG: Influenza in hospitalized adults: gaining insight into a significant problem. J Infect Dis 2009;200:485-488. 
33 Birnkrant D, Cox E: The emergency use authorization of peramivir for treatment of 2009 H1N1 influenza. N Engl J Med 2009; 361:2204-2207.

34 World Health Organization: Antiviral use and the risk of drug resistance. Pandemic (H1N1) 2009 briefing note 12; 29 September, 2009. http://www.who.int/csr/disease/swineflu/notes/h1n1_antiviral_use_20090925/ en/index.html (cited February 11, 2010).

35 Fiore AE, et al: Prevention and control of seasonal influenza with vaccines: recommendations of the Advisory Committee on Immunization Practices (ACIP), 2009. MMWR Recomm Rep 2009;58(RR-8):1-52.

$>36$ Govaert TM, et al: The efficacy of influenza vaccination in elderly individuals. A randomized double-blind placebo-controlled trial. JAMA 1994;272:1661-1665.

37 Rivetti D, et al: Vaccines for preventing influenza in the elderly. Cochrane Database Syst Rev 2006;3:CD004876.

38 Jefferson T, et al: Efficacy and effectiveness of influenza vaccines in elderly people: a systematic review. Lancet 2005;366:1165-1174.

39 Ahmed AE, Nicholson KG, Nguyen-VanTam HS: Reduction in mortality associated with influenza vaccine during 1989-90 epidemic. Lancet 1995;346:591-595.
40 Voordouw AC, et al: Annual revaccination against influenza and mortality risk in community-dwelling elderly persons. JAMA 2004;292:2089-2095.

41 Simonsen L, et al: Impact of influenza vaccination on seasonal mortality in the US elderly population. Arch Intern Med 2005; 165 : 265-272.

42 Kelly H, Vu T, Smith D: Influenza vaccination and mortality in the United States. Arch Intern Med 2005;165:2037-2038; author reply 2039-2040.

43 Thompson WW, et al: Influenza vaccination among the elderly in the United States. Arch Intern Med 2005;165:2038-2039; author reply 2039-2040.

-44 Holland D, et al: Intradermal influenza vaccine administered using a new microinjection system produces superior immunogenicity in elderly adults: a randomized controlled trial. J Infect Dis 2008;198:650 658.

45 Chi RC, Rock MT, Neuzil KM: Immunogenicity and safety of intradermal influenza vaccination in healthy older adults. Clin Infect Dis 2010;50:1331-1338.

46 Iob A, et al: Evidence of increased clinical protection of an MF59-adjuvant influenza vaccine compared to a non-adjuvant vaccine among elderly residents of long-term care facilities in Italy. Epidemiol Infect 2005;133: 687-693.

-47 Falsey AR, et al: Randomized, double-blind controlled phase 3 trial comparing the immunogenicity of high-dose and standarddose influenza vaccine in adults 65 years of age and older. J Infect Dis 2009;200:172-180.
48 Centers for Disease Control and Prevention (CDC): Licensure of a high-dose inactivated influenza vaccine for persons aged $>$ or $=65$ years (Fluzone High-Dose) and guidance for use - United States, 2010. MMWR Morb Mortal Wkly Rep 2010;59:485-486.

49 Weycker D, et al: Population-wide benefits of routine vaccination of children against influenza. Vaccine 2005;23:1284-1293.

50 Ghendon YZ, Kaira AN, Elshina GA: The effect of mass influenza immunization in children on the morbidity of the unvaccinated elderly. Epidemiol Infect 2006;134:71-78.

51 Reichert TA, et al: The Japanese experience with vaccinating schoolchildren against influenza. N Engl J Med 2001;344:889-896.

52 Carman WF, et al: Effects of influenza vaccination of health-care workers on mortality of elderly people in long-term care: a randomised controlled trial. Lancet 2000;355: 93-97.

53 Vajo Z, et al: Safety and immunogenicity of a 2009 pandemic influenza A H1N1 vaccine when administered alone or simultaneously with the seasonal influenza vaccine for the 2009-10 influenza season: a multicentre, randomised controlled trial. Lancet 2010; 375:49-55.

54 Perez-Padilla R, et al: Pneumonia and respiratory failure from swine-origin influenza $\mathrm{A}$ (H1N1) in Mexico. N Engl J Med 2009;361: 680-689. 